\title{
LA EDUCACIÓN AMBIENTAL \\ EN EL PROYECTO EDUCATIVO INTEGRAL \\ COMUNITARIA: UNA REFLEXIÓN \\ DESDE LA PARTICIPACIÓN EN \\ COMUNIDADES ESCOLARES
}

\section{Yamarú del Valle Chirinos Araque}

Doctora en gestión de la innovación, Universidad Dr. Rafael Belloso Chacín. Investigadora Asociada categorizada por Colciencias. Docente- investigadora del grupo: GORAS. Universidad Católica Luis Amigó, Medellín - Colombia: yamaru.chirinosar@ amigo.edu.co ORCID ID. 0000-0003-0471-9859.

\section{Coromoto González}

Doctora en innovaciones educativas, UNEFA, Especialista en Metodología de la Investigación, Psiconeurolingüista y Psiconeuroendocrinologia. Terapeuta Familiar. Profesora asociada en la UNERMB.

\section{Víctor Hugo Meriño Córdoba}

Doctor en Educación, Universidad Dr. Rafael Belloso Chacín. Investigador Senior, categorizado por Colciencias. Docente investigador del grupo: GORAS. Universidad Católica Luis Amigó, Medellín - Colombia, victor.merinoco@amigo.edu.co ORCID ID. 0000-0001-8911-7202

\section{Carmen Ysabel Martínez de Meriño}

Doctora en Educación, Universidad Dr. Rafael Belloso Chacín. Investigadora Asociada categorizada por Colciencias. cimartinezunermb@gmail.com ORCID ID.0000-0002-8134-2306

\section{Resumen}

El propósito de esta investigación fue determinar el nivel de participación de la comunidad en el proyecto educativo integral comunitaria que aborda la educación ambiental en las instituciones de educación básica del municipio Maracaibo, sustentado en los aportes de Rivarosa (2005), Casilla (2002), Benayas, Gutiérrez \& Hernández (2003), entre otros. la investigación fue descriptiva, 
con diseño no experimental, transversal. la población estuvo constituida por 924 individuos, entre directivos, docentes, personal administrativo, obreros, padres, representantes y miembros de la comunidad, con una muestra de 213 sujetos la información se recabó aplicando tres cuestionarios, validados por expertos y sometidos a la confiabilidad a través del coeficiente alfa cronbach, extrayendo distribución de frecuencias para el análisis de los datos. los resultados obtenidos permiten concluir que la participación de la comunidad escolar presenta un nivel moderado en las actividades para la preservación ambiental y en el proyecto educativo integral comunitaria; recomendando generar una comunicación efectiva y la autogestión con los miembros de la comunidad educativa para el logro de una educación ambiental proactiva y dinámica.

Palabras clave: participación, comunidad, proyecto educativo integral comunitario, educación ambiental

\section{ENVIRONMENTAL EDUCATION IN THE COMMUNITY INTEGRAL EDUCATIONAL PROJECT: A REFLECTION FROM PARTICIPATION IN SCHOOL COMMUNITIES}

\section{Abstract}

The purpose of this research was to determine the level of participation of the community in the educational integral community project that addresses environmental in basic education in the maracaibo municipality institutions education based on the contributions of rivarosa (2005), box (2002) and benayas, gutierrez and hernández (2003), among others. research was descriptive field, with no experimental, cross design. the population was made up of 924 individuals between managers, teachers, administrative staff, workers, parents, representatives and members of the community, with a sample of 213 subjects information was sought by applying three questionnaires, validated by experts and undergoing reliability on the alpha cronbach coefficient, extracting distribution of frequencies for data analysis. the results allow you to conclude that the participation of the school community presents a level moderate on activities for environmental preservation and in the community comprehensive educational project, recommending generate effective communication and self-management with members of the 
educational community towards a dynamic and proactive environmental education.

Keywords: participation, community, project educational integral community, environmental education

Artículo resultado de investigación, con un enfoque cuantitativo, resultado de un proyecto de investigación culminado.

\section{Introducción}

Actualmente, es de vital importancia hablar acerca de la educación ambiental como punto de partida para la preservación y conservación de los espacios y/o recursos naturales, en función de la situación experimentada por la sociedad general en cuanto a la crisis ambiental, y en ese ámbito específico, la educación como agente de cambio puede generar acciones de carácter conceptual, procedimental y actitudinal, que propicien en el hombre, la construcción de conocimientos que lo lleven a cuidar y proteger el medio ambiente donde se desenvuelve, con miras de mejorar su calidad de vida, logrando el mantenimiento efectivo del entorno que le rodea. Cabe destacar que la crisis ambiental según los estudios realizados por Teitelbaum (1978), Carrasco (1996), entre otros, la ha ocasionado el hombre, quien al darle paso a los avances científicos, tecnológicos y de la informática, por esa competitividad latente y permanente que mantiene, crea, inventa, e innova procesos y productos, que en el tiempo, por su acción, han ido destruyendo el medio natural donde se desenvuelve, por lo cual, se dice que una de las razones de esta situación, es la falta de conciencia del hombre sobre su medio, quien creyéndose dueño, lo ha afectado, sin considerar la ley del retorno y por eso, él mismo, recibe los efectos negativos que se manifiestan en el medio ambiente.

Así mismo, Smith (1997), expresa que los problemas ambientales son frecuentemente causados por las sociedades humanas, las cuales son colectividades de individuos. Por lo tanto, los individuos resultan ser las causas primarias de muchos problemas, y la solución a los problemas probablemente será el individuo (actuando colectivamente). En ese orden de ideas, la Educación ambiental, 
relaciona al hombre con su medio ambiente, con su entorno y busca un cambio de actitud, una toma de conciencia sobre la importancia de conservar para el futuro y para mejorar nuestra calidad de vida, considerando un proceso de aprendizaje donde se promueva la participación de todos los actores involucrados en los problemas de una comunidad, lo cual implica despertar conciencia, comprender y analizar las situaciones del medio y promover comportamientos dirigidos al desarrollo de actitudes y habilidades prácticas que mejoren la calidad de vida, como lo expresa Abreu (1996).

En el campo educativo, la Educación Ambiental, es materia y tema de carácter obligatorio, pero posiblemente habría que decir que se ha quedado allí, en lo teórico porque en la práctica, son pocos los cambios detectados. Benayas, Gutiérrez \& Hernández (2003), expresan que las intervenciones educativas no son de gran utilidad si no van acompañadas de medidas de gestión eficaces. En bastantes ocasiones las campańas de sensibilización ambiental generan la necesidad de un comportamiento específico sin que existan las infraestructuras apropiadas para responder a estas nuevas demandas.

Al respecto, en Venezuela, se constata que los esfuerzos han sido muchos, pero desde el punto de vista educativo, los trabajos han sido teóricos desde la escuela y más directamente en el aula, donde el docente desarrolla la temática y enfatiza en el contenido, hechos que a niños, nińas y adolescentes, deja mucho para la reflexión, pero en la práctica, en el desenvolvimiento cotidiano, no son relevantes los cambios que se han logrado y por ello, el medio ambiente está cada vez más deteriorado, afectando la salud, el crecimiento y desarrollo de los ciudadanos, de allí, la divergencia entre lo teórico y lo práctico, porque lo que se dice no se evidencia en su aplicación. Ante estos contextos, el problema de la crisis ambiental es abordado en los proyectos realizados con la comunidad educativa asumiendo determinadas conductas como reciclar residuos, aunque no se les suministra el soporte necesario para llevar a cabo estas actividades por diferentes razones (los contenedores no existen, están repletos, no se reciclan realmente, entre otros), en fin, el proceso se queda en lo teórico, y no se logra la participación esperada de los ciudadanos.

Cabe decir, no es un problema exclusivo de Venezuela, es a nivel mundial, de allí la necesidad de responder a interrogantes al respecto de por qué los ciudadanos son indiferentes ante las realidades 
que se observan en su medio ambiente y cuáles acciones ejecutan para darle respuesta a éstas ¿será que harán cambios de conducta, y asumirán una actitud de interés ante éstas? ¿Qué es lo que está sucediendo con las actividades de Educación Ambiental? La intención y el logro de la eficacia educativa generan con toda seguridad la exigencia de una participación comunitaria que responda a procesos de interacción para la formulación y ejecución de diferentes actividades, mediante una especial concepción de la ciudadanía y del sentido de adhesión y pertenencia a una comunidad. Se trata, en resumen, de una intermediación de las instituciones educativas en las iniciativas sociales, haciéndose responsables como principio de compromiso compartido con los integrantes de la comunidad y de la escuela, participando en la elaboración, ejecución, control y evaluación del Proyecto Educativo Integral Comunitario dirigidos a preservar el medio ambiente.

Tal planteamiento, enfatiza en la necesidad de establecer grandes cambios que no serán posibles sino a través de una amplia interacción sinérgica y del consenso entre todos los actores involucrados en el hecho educativo, tendientes a acelerar un desarrollo y por ende, mejorar su calidad, actuando de manera responsable y comprometida en la preservación de su medio ambiente, aportando no solo los aspectos teóricos referidos a la Educación Ambiental, sino que se formulen estrategias de cambio para el desarrollo sostenible. Todo esto debe expresarse en un contexto de permanente reciprocidad entre las escuelas y las comunidades donde ellas están inmersas, prevaleciendo las buenas relaciones humanas, la exaltación de los valores, el trabajo en equipo, el cambio de actitudes, la confianza, la visión compartida, la trascendencia de la información; todo lo cual conlleva a un cambio de conducta que facilita la solución a los diversos problemas que confronta el medio ambiente que circunda las escuelas y no son resueltos muchas veces, posiblemente por la apatía y el poco compromiso que demuestran los actores de estas organizaciones.

Es necesario entonces, el convencimiento para lograr fórmulas que transformen la participación que hasta ahora pareciera no haber respondido a la realidad de las instituciones educativas; por ello, las escuelas y sus comunidades deben redefinir sus roles abriendo espacios para la reflexión e integración, traducida en pensamientos 
y acciones de identidad colectiva que contribuyan con la formación de los ciudadanos para relacionarse adecuadamente con su medio ambiente, lo cual exige el mayor esfuerzo como forma de mejorar las capacidades individuales y colectivas, organizando las comunidades, provocando en ellas las transformaciones que los colectivos y su entorno necesitan.

La propia Constitución de la República Bolivariana de Venezuela (1999), reconoce el derecho y el deber que tienen todos los ciudadanos a participar libremente en los asuntos públicos como forma de garantizar su completo desarrollo; promocionándolo de manera local, sustentable y sostenible. Vista así, la educación debe estar enmarcada en los principios expresados en la Carta Magna; con una visión humanista, constructivista, centrada en principios de igualdad, justicia, libertad y equidad social, a través de la cual se rescate la construcción en colectivo; contando con las posibilidades y potencialidades de sus actores, considerando la diversidad de culturas, costumbres, horarios, donde las escuelas sean el centro del quehacer comunitario, actuando como espacio preservador y orientador del ambiente, impulsor del desarrollo local sustentable, donde el ser humano sea el protagonista.

De esa manera, la institución educativa se abre a la posibilidad de extender su acción a la comunidad y a la sociedad, en vez de centrarse solamente en la instrucción de niños, niñas y adolescentes, hecho que puede alcanzarse a través del desarrollo del Proyecto Educativo Integral Comunitario, donde se impulsa la metodología participativa. Sin embargo, en documentos del Ministerio de Educación, Cultura y Deportes (2001), se afirma que la escuela y la comunidad siguen divorciadas, pretendiendo por esto que, a través del Proyecto Educativo, se fijen los lineamientos en relación a la integración y participación de la comunidad. Al respecto, la educación básica y todo el sistema educativo deben considerar a la comunidad como el ambiente general que rodea a la escuela considerándose un componente del micro-sistema social de la comunidad local al cual debe la escuela integrarse.

De esta manera, se entiende que la acción participativa será posible en la medida que se fortalezca el liderazgo compartido, reforzando el carácter democrático de la actividad comunitaria, enmarcada con un espíritu cooperativista, crítico, reflexivo 
y autogestionario, capaz de descubrir a los líderes potenciales que existen en la comunidad para que enlacen y concentren los esfuerzos con sentido colectivo en la realización del trabajo comunitario. Por consiguiente, se observa que en la educación se produce un tipo de actividad social que exige la participación, en tanto, la actuación educativa se ejerce en nombre de los intereses generales de la comunidad, como por deseo expreso de los principales responsables de la educación de jóvenes y niños; es decir, los padres y representantes. La educación incide sobre la personalidad individual, sobre los valores sociales y sobre el conjunto de la cultura de una comunidad. Indudablemente que, pasar de no permitir siquiera opiniones y abrir todos los espacios de decisión, puede resultar desconcertante y hasta contraproducente, sin contar que los propios actores del proceso educativo no tienen, en la mayoría de los casos, experiencia de la real participación en las decisiones sobre la organización, su proyecto y su ejecución.

Las leyes venezolanas pueden alentar, impulsar la participación de la comunidad en los problemas del medio ambiente, legitimarla, lo cual es significante; pero no la garantizan. Es necesario planear, según el caso, qué tipo de participación, en qué aspectos, a través de qué mecanismos, en qué procesos, quiénes participan y cuáles serán las responsabilidades; expresando Alfiz (2003), que esto da respuesta oportuna y compatible a la propuesta de escuela que se quiere. Entonces se podría hablar, de una cultura de participación educativa como la acción de intervenir en aquellas situaciones escolares de carácter colectivo que vienen condicionadas por el conjunto de creencias, valores, opiniones y actitudes de un individuo o grupo referidas tanto al concepto de educación ambiental como al propio de participación de las comunidades.

La simple convocatoria no significa espacio de participación real, en ocasiones incluso, puede resultar una forma engañosa de legitimar decisiones elaboradas previamente, la cual cuando es comunitaria implica organizarse como grupo; los vecinos de la escuela no son necesariamente actores de la comunidad, se convierten en eso cuando se organizan e intervienen en la vida comunitaria. Lo mismo ocurre con los padres y representantes, quienes participan individualmente en relación con los hijos, en el proceso enseñanza aprendizaje, pero esta participación se hace colectiva en la 
medida que se hacen actores de las organizaciones que conforman la estructura funcional de la escuela.

Esta es la problemática que se presenta en las Escuela Básicas Nacionales donde el personal directivo, docente, administrativo y obrero de la institución, brinda poca oportunidad a la comunidad educativa así como tampoco a los sectores o fuerzas vivas de la localidad, para participar, además, el PEIC pocas veces se dirige a la preservación del medio ambiente, lo cual implica que las ideas, opiniones y decisiones surgen siempre del personal del plantel, tomando solo a los padres y representantes y miembros de la localidad para informarles aspectos que muchas veces son de interés para la escuela, o para solicitarles colaboración, lo cual en ocasiones es obviado al no estar identificados con la visión, misión y objetivos de la institución.

Por esta situación, los padres, representantes y miembros de la comunidad manifiestan desinterés, apatía e indiferencia por todo aquello que ocurre en la escuela, lo cual afecta las condiciones de interacción e integración que debe establecerse entre la escuela y la comunidad, así como se evidencia que las acciones en las cuales deberían participar padres, docentes y comunidad, no se hacen de esa manera, como es el caso de la planificación, ejecución y evaluación de la Educación Ambiental abordado en el PEIC, donde sólo participan los docentes de la institución comisionados para la actividad sin dar oportunidad a la asamblea así como los estudiantes, de conocer, opinar, aprobar o desaprobar los hechos planteados en el proyecto.

Por ello, es necesario activar la educación ambiental con una participación consciente, comprometida y efectiva; generadora de soluciones para el colectivo al brindar nuevas formas de abordar tanto la realidad cotidiana del hecho educativo como de la compleja y dinámica realidad, considerando la participación de la comunidad para el desarrollo del PEIC, dirigido a preservar el medio ambiente local, regional y nacional. Estos planteamientos llevan a formular la siguiente la interrogante: ¿Cuál es el nivel de participación de la comunidad educativa en el Proyecto Educativo Integral Comunitario que aborda la Educación ambiental en las instituciones de Educación Básica del Municipio Maracaibo? Partiendo de la reflexión que surja de las opiniones aportadas por el personal 
directivo, docentes, administrativo, obreros, padres/representantes y comunidad en general. La situación descrita, demanda formular como objetivo general de este estudio, determinar el nivel de participación de la comunidad en el Proyecto Educativo Integral Comunitario que aborda la educación ambiental en las instituciones de Educación Básica del Municipio Maracaibo.

\section{Fundamentación teóricos}

La educación ambiental, se convierte en una tarea interesante que actualmente preocupa a los investigadores del sector educativo por cuanto se plantea la necesidad de darles corresponsabilidad a los padres, representantes y comunidad en general, así como al personal docente y directivo en el hecho educativo para la preservación $\mathrm{y}$ adecuado uso del medio ambiente, asumiendo su participación en el Proyecto Educativo Integral Comunitario.

\section{Educación Ambiental}

Cuando se habla de educación ambiental, se está determinando una acción que conjuga la educación como agente de cambio y el medio ambiente donde el hombre se desenvuelve, y por ello, Teitelbaum (1978: 51), planteó que "es la acción educativa permanente por la cual la comunidad tiende a la toma de conciencia de su realidad global, del tipo de relaciones que las personas establecen entre sí y con la naturaleza”, así como supone también la manera cómo actúa para darle solución a los distintos problemas que surgen, producto del comportamiento, de los valores y la actitud hacia la realidad circundante. Desde esa perspectiva, se estudia la Educación ambiental, en la escuela, bajo el enfoque pedagogista y crítico, que como lo explica Rivarosa (2005), plantea el cambio, utilizando el medio como recurso para el desarrollo del individuo, haciendo uso de las posibilidades educativas del entorno natural, de manera que se eduque desde el medio, así como por y para el medio, de manera que críticamente, surja un visión integral del ambiente socio natural, generándose un compromiso de la comunidad para mejorar las condiciones de convivencia y preservación del medio ambiente.

Por esta razón, desde la escuela con la comunidad, deben realizarse proyectos de aprendizaje e integrales que se dirijan a solventar 
los distintos problemas que a nivel ambiental se presentan y afectan el bienestar de los niños, nińas, adolescentes y comunidad en general, de allí que sea responsabilidad de todos, trabajar para el cambio y la transformación teórica y práctica, así como actitudinal, involucrando procesos de reflexión acerca de la participación de la comunidad para el desarrollo de los proyectos encaminados a la Educación ambiental, intentando favorecer las interacciones culturales, y socio educativas.

Carrasco (1996), resalta que es importante tener en cuenta los avances de algunos docentes e investigadores quienes a propósito del constructivismo, elaboran conocimientos en torno a la comprensión de los problemas ambientales relacionados con contextos naturales, sociales y culturales, al partir de la exploración de criterios y conceptos, identificando la realidad circundante a la escuela, sensibilizando a estudiantes, padres, representantes, organismos públicos y privados, así como a todo el personal de la institución, para que se dinamicen vean, sientan y reconozcan lo que tienen y lo que hace falta. En torno a este aspecto, se deben diseñar proyectos factibles referidos a la Educación ambiental, en este caso el PEIC, comprometiéndose en enriquecer los saberes conceptuales, procedimentales y actitudinales, que le permitan a la comunidad educativa, la comprensión de su propia realidad y coadyuve a la generación de estrategias de autogestión y cogestión con la participación comunitaria y ciudadana para la búsqueda de soluciones a la crisis ambiental que se experimenta en las localidades y en especial en la región zuliana.

\section{Proyecto Educativo Integral Comunitario}

El proyecto educativo comunitario (PEIC), según Morales (2006), es un proyecto, concebido como proceso de planificación personal o grupal, acorde a las características propias de los involucrados en contextos y situaciones específicas, en el cual puede haber asesoría externa, generando el compromiso inherente a la construcción, secuencial y sistémica, que lleva a la concreción de un ideal individual y colectivo. Es educativo, porque toda acción del proyecto lleva la intencionalidad del logro de aprendizajes significativos y estratégicos, que pueden ser formales o no y desarrollarse en variedad de espacios y a través de diferentes actividades. Es integral, porque 
parte y trabaja por la unidad, por el desarrollo del sujeto y del colectivo en su totalidad holística y ecológica. Implica conjunción de fuerzas, es interdisciplinario, no parcelado.

Es comunitario, ya que es un esfuerzo dirigido a la comprensión humana del quehacer grupal, a la conciencia común, a la formación y dirección colectiva, a normas comunes de proceder y obrar, es decir, a la creación de la ciudadanía como sentir, expresión, hecho y resultado social. Su trabajo, se fundamenta en una filosofía humanista de participación plena, donde el colectivo institucional y comunitario se constituyen en sujeto protagónico; los problemas de la comunidad se convierten en el eje del quehacer escolar y la institución educativa en el centro que promueve la formación permanente, la participación y organización comunitaria. El PEIC, en el escenario de la institución educativa, tiene como fundamento la formación integral del sujeto-ciudadano, como artífice de su propio desarrollo, en el contexto de una democracia participativa y protagónica con equidad social. Este enfoque de la nueva escuela comunitaria trasciende la participación, como consenso hacia la humanización de la corresponsabilidad y solidaridad de todos los actores involucrados en el quehacer educativo para lograr el bienestar social.

Este deber ser, enfatiza en tres dimensiones del PEIC: pedagógica y andragógica, gestión administrativa y participación comunitaria. la dimensión pedagógica andragógica pretende la promoción y facilitación de los aprendizajes a través de formación ciudadana integral en y para la vida, liderazgo efectivo, democratización del saber, adecuación del currículo a las características de los educandos y del entorno local del plantel para el desarrollo endógeno, calidad de la práctica pedagógica y andragógica, diseño de la planificación educativa entre todos los actores, actividades con sentido personal social que se evidencien en producciones variadas se conecta con otras similares, proyectándose y retroalimentándose, así como producción de recursos para el aprendizaje cooperativo.

En cuanto a la gestión organizacional, se propone un Modelo de gerencia participativa no de una persona o equipo gestor, aplana las jerarquías: planificación, ejecución y evaluación, con la gestión dialógica en relaciones de igualdad entre los actores, corresponsabilidad en las decisiones, adecuando los procedimientos de la dinámica de la escuela (horarios, turnos, matrícula) a los requeri- 
mientos y necesidades de la diagnosis de la realidad local y realidad educativa. De igual manera, desarrolla la gestión, control social y uso racional de recursos institucionales, humanos, materiales y financieros, propiciando calidad de las relaciones interpersonales, con la creación de los Consejos Parroquiales y Municipales de educación como mecanismos de corresponsabilidad y participación protagónica de las comunidades y la sociedad civil. Hace uso del trabajo cooperativo en redes.

La dimensión comunitaria: manifiesta la disposición y conciencia social con la visión de lo que conviene a todos, donde se busca que la comunidad sea el eje del quehacer escolar. Por ello promueve la calidad de las relaciones de la escuela con los padres y representantes y demás miembros del contexto local comunitario, considerando al plantel un espacio comunitario, vinculando a las instituciones educativas a los planes de desarrollo local, municipal, estadal, nacional, latinoamericana, de manera que se incrementen los niveles de participación en la solución de conflictos que presenta la escuela y la comunidad. Por esto, se da apertura del espacio o escenarios que le oferta la escuela a las actividades de las asociaciones civiles, de padres y representantes y demás actores del proceso educativo comunitario, haciendo énfasis en el desarrollo sostenible para la preservación y uso óptimo de los espacios y sus recursos, generando influencia escolar en la acción a la familia y comunidad, además de fortalecer las instituciones educativas como espacios comunitarios, a través de la promoción hacia la organización comunitaria a través de los consejos comunales, consejos parroquiales consejo local de planificación pública.

En ese orden de ideas, se detecta que el PEIC, debe estar cristalizado en la fusión de estas tres dimensiones y en la concreción de la formación del ciudadano en situación de plena intervención en la gestión local, donde la Institución educativa facilite el protagonismo de gestión social comunitaria. Expresa Bacalini (1998), que las redes sociales nacieron para quedarse con el hombre, con la sociedad: deben ser entendidas como sistemas abiertos, mediante los cuales se produce un intercambio dinámico tanto entre sus propios integrantes como con integrantes de otros grupos y organizaciones, posibilitando las potencialidades de los recursos que poseen, por lo cual se sugiere redimensionar el concepto de lo ecológico: 
naturaleza de la gestión ambiental, que constituye el conjunto de actividades, funciones, organización, recursos, instrumentos de política y sistemas de participación, aplicados a la administración de los bienes naturales que integran nuestro entorno, que incluye la sociedad y sus relaciones con la naturaleza, y que buscan adaptar las actividades humanas a las capacidades y limitaciones del ambiente natural y humano.

El desafío de la educación tiene que ver con la propuesta del desarrollo sostenible y sustentable, armónico, que propicie expectativas de superación de la pobreza hacia el bienestar social con calidad de vida. Lograr un equilibrio en lo económico, que fomente la cultura al trabajo y la productividad, que siembre el petróleo en el agro, para alcanzar la seguridad alimentaria; esta es una tarea que requiere transformación de los programas educativos y de los currículos; así como potenciar la riqueza humana aprovechando los recursos existentes. En resumen, deben prevalecer los valores de justicia, independencia, igualdad, libertad, paz y autodeterminación, participación protagónica, democratización del saber, cooperación, solidaridad, convivencia, bien común, unidad e integración, corresponsabilidad, contraloría social, por lo cual se fundamenta en el fortalecimiento de la cooperación internacional, soberanía nacional en un mundo multipolar para ser más equitativo el proceso de toma de decisiones en la lucha contra la pobreza, el desarrollo sustentable y la integración latinoamericana y caribeña para la instrumentación de la identidad común: crecimiento, desarrollo y bienestar social.

\section{Participación en comunidades escolares}

La educación es un hecho definitivamente humano que le facilita a cada persona los medios para modelar libremente sus cualidades y capacidades creativas, ya que tiene como misión promover el desarrollo continuo de la persona, responsabilizándose de sí misma y participando en los diferentes contextos donde se desarrolla su existencia. De forma figurada, se compara la participación con un crisol, por lo cual Santos (1998), explica esto al plantear que es un crisol de pensamiento porque se promueve y desarrolla el debate y la discusión, donde se conjugan voluntades tomando decisiones de forma compartida y no autoritaria, considerando sentimientos al establecer la comunicación y la percepción emocional de la reali- 
dad, con ella todos pueden comunicarse y percibir emocionalmente el modo de vivir, la realidad educativa y las acciones que confluyen en iniciativas de los integrantes de la comunidad.

Por su parte, Ander (2001), afirma que se trata de un acto ejercido por un sujeto/agente que tiene una presencia activa en aspectos que conciernen a su vida, ya sea en lo personal, familiar, laboral o en relación con su entorno. Asimismo, la participación es un valor en la comunidad educativa, porque encierra en su misma naturaleza, divisiones democráticas, posibilidades de aprendizaje y vivencias de comunicación. Participar es aportar conocimientos a la tarea compartida de construir y reconstruir los saberes, intervenir en las decisiones, controlar los procesos, intercambiar las experiencias. Esto implica una forma de acción y de compromiso para alcanzar algo en común, ya que participar es siempre un actuar con otros o en relación con otros. Sea cual fuere el campo de la participación, descansa sobre unos principios básicos que son la condición sine qua non para que ésta sea real, de allí que no es algo que se conceda, tiene que ver con el derecho de toda persona, colectivo o pueblo a poder intervenir en las decisiones que afectan su vida, que inciden sobre su destino personal y colectivo. Es expresión de la responsabilidad y libertad del ser humano para hacer su propio destino sin delegar en otros esa tarea. Hacer uso del derecho a participar potencia a la persona.

La participación no es abstracta, sino en algo y para algo que se relaciona con los intereses y/o valores de la persona que la practica; nadie participa sin saber para qué, dónde, cómo y con quién. De poco sirve la participación en sí, que no sea para algo concreto, ya sea el proceso productivo, del espacio en que vive, de la vida política, de la sociedad civil, de servicios que se prestan o se reciben. El proceso de participación en las organizaciones es uno de los fenómenos sociales menos comprendidos. Esta incomprensión probablemente se debe a que se trata de un proceso complejo y sutil. También es destacable la diferencia de significados sobre participación, según el marco teórico y de valores donde se aplique, de esta forma, unos inciden más en los aspectos sociales y otros en los organizativos; que unido a los aspectos psicológicos y sociológicos que intervienen, configuran un panorama conceptual realmente complejo. No obstante, las apreciaciones de Davis \& Newstrom (2004: 246) acercan a una definición: 
La participación es un elemento importante que contribuye a la eficiencia organizacional. La participación es el involucramiento mental o emocional de las personas en situaciones de grupo, que las estimula a colaborar en la obtención de las metas del grupo y a compartir la responsabilidad de ellas. Para los empleados es el resultado psicológico de la administración de apoyo. En todo caso, parece claro el carácter instrumental que adquiere la participación en la dinámica organizativa y de funcionamiento en los centros escolares al servicio de la actividad educativa, incluso de los fines del propio sistema ambiental, pues permite llegar a conclusiones importantes en cuanto a los distintos modos de actuar en las organizaciones.

Para Geilfus (2005), la participación tiene una escala que va de la pasividad, cuando solo se informa a las personas, el suministro de información, respondiendo la gente a través de encuestas, sin posibilidad de influir, participando por consulta si se escuchan los puntos de vista pero esto no tiene incidencia en las decisiones, así como la participación por incentivos, suministrando principalmente trabajo u otros recursos a cambio de ciertos incentivos (materiales, capacitación, sociales, económicos), pero tampoco esto influye en las decisiones que los superiores tomen, además de mencionar el autor citado, la participación funcional, si las personas forman grupos de trabajo para responder a objetivos predeterminados .

De igual manera, se considera en esta escalera, la participación interactiva, porque la gente actúa directamente en el análisis y definición de las acciones, en la formación y fortalecimiento del proceso, los grupos controlan las decisiones locales y se mantienen las estructuras y prácticas, así como plantea Geilfus (2005), el autodesarrollo ocurre cuando los grupos de manera organizada toman las decisiones con iniciativa, sin esperar intervenciones externas, lo cual evidencia la capacidad de la gente para involucrarse en las actividades de la escuela. En consecuencia, la participación contribuye a desarrollar la responsabilidad y la capacidad de dialogar, de escuchar, de planificar, de evaluar, de aprender y de trabajar en equipo, es comprometerse crítica y constructivamente en una tarea colectiva.

En este sentido, solo se puede llegar a vivenciar estos principios y derechos cuando el individuo ha conseguido interiorizarlos como integrante de una colectividad que asume cotidianamente las estructuras democráticas y los valores que en ellas subyacen, tales 
como la aceptación de las decisiones mayoritarias, el respeto a las opiniones disidentes de la minoría, el valor del diálogo como método para superar los conflictos y la representatividad de los representantes elegidos democráticamente. Según Viñas \& Doménech (1998), la participación no es ninguna moda, es un principio que en educación se fundamenta en niveles muy diversos, que se detallan a continuación:

Educativo: aumenta la calidad de la educación, es decir, favorece la implicación de los actores en el proceso educativo. Participación y educación son dos elementos inseparables. La educación debe ser participativa, y la participación constituye una forma de educación.

Social: la escuela es una institución de carácter social en la que los ciudadanos tienen derecho a participar, en este sentido, la educación es una labor compartida, se parte de la premisa que los agentes educativos son diversos, así como diversa es su responsabilidad y su papel.

Profesional: la enseñanza es una labor de equipo, mediante la cual se mejora la calidad y la profesionalidad de sus actores, es decir, se hace participativo. Es preciso ponerse de acuerdo e intercambiar ideas y promover reflexiones sobre la propia práctica y sobre el funcionamiento de la institución escolar, que no se podrá hacer de forma individual, ni tampoco con una estructura vertical.

El Estado venezolano consciente de esta realidad ha planteado, entre otros caminos, la posibilidad de transferir competencias político-administrativas a las regiones y comunidades, lo que ha denominado como descentralización de la educación para hacer más eficientes un conjunto de acciones gerenciales técnico-administrativas y pedagógicas tendentes a fortalecer la gestión autónoma de los planteles y generar procesos de participación más efectivos, todo ello enmarcado en el Proyecto Educativo Nacional (Ministerio de Educación, Cultura y Deportes, 2001), donde la participación de todos los actores es el eje central del éxito escolar, llevándose a cabo a través de los proyectos y de la metodología participativa.

En este orden de ideas, los aspectos Pospositivos del Proyecto Educativo Nacional (MECD, 2001), consideran promover los enfoques organizativos y una nueva gerencia participativa; donde se plantea la descentralización como la vía para la participación y au- 
tonomía real de los actores involucrados en el proceso educativo y lograr así una sociedad sostenible donde se propicie el acercamiento de las comunidades y la toma de decisiones. Según el documento, los cambios que demandan las escuelas básicas, ubica a la comunidad local y a la escuela como protagonista de una toma de decisión académicamente pertinente. De allí, que la participación apoya la gestión comunitaria dentro de ciertos lineamientos que suman compromisos y disposición hacia la cohesión donde debe dársele cabida a la educación Ambiental tanto formal como informal.

En tal sentido, los límites de la participación coinciden con los límites de las competencias de cada persona y su disponibilidad para actuar, correspondiéndole a la gerencia de las escuelas, motivar y propiciar situaciones donde participe la comunidad en pro de la preservación del medio ambiente y todo aquello que propenda a mejorar la calidad de vida. Por consiguiente, en las organizaciones educativas ningún docente o representante, o empleado puede ser el único responsable de todos los resultados; necesariamente todo esfuerzo es del equipo y todo resultado es producto del equipo, relacionándose constantemente.

Para Chiavenato (2003), la práctica de las relaciones interpersonales consiste en establecer o mantener contacto con otros individuos. Significa estar condicionado en las relaciones por una actitud, un estado de espíritu o una manera de ver las cosas, que permita comprender a las otras personas y respetar su personalidad, cuya estructura, es sin duda, diferente. En consecuencia, es indispensable realizar los esfuerzos tendientes a establecerlas, por ello, en las organizaciones escolares debe existir una buena comunicación y coordinación de los esfuerzos para lograr y mantener un clima positivo y favorable que permita un sustancial aumento de la productividad en las mismas.

Dentro de este orden de ideas, las instituciones educativas como organizaciones sociales, están formadas por personas que se interrelacionan con el fin de alcanzar propósitos comunes por medio de la cual fluye la información que sirve para transmitir las metas, objetivos, estrategias y actividades que dirijan la línea de la organización, con ella, los miembros van a estar conscientes de los roles que debe3n cumplir, asumiendo las funciones y tareas de la mejor manera para desarrollarlas más fácilmente, es decir, el personal au- 
nará esfuerzos para ejecutar tareas en conjunto, donde la empatía entre los miembros es el medio fundamental que lleva a comprender y ayudar a las otras personas en sus tareas, cuando lo ameriten. Para analizar la participación comunitaria se requiere establecer los fundamentos del enfoque de la comunidad en cuanto a su participación en la escuela, así como estrategias teóricos-prácticas que la favorezcan en el hecho educativo, considerando que el medio social, cultural (sus instituciones y grupos humanos) ejercen influencia en la orientación y administración de las instituciones escolares, éstas a su entorno, al medio ambiente, ejerciendo influencia de retorno sobre los grupos sociales, las personas y las instituciones del medio social, produciendo así, un movimiento cíclico que determina cambios en la estructura y concepción cultural de las sociedades, por ello, la comunidad como unidad social básica, comparte el área de convivencia mutua, con una serie de factores de común interés, anhelos o funciones que se les imponen más intensamente que otras circunstancias accidentales.

Con base a esta definición, las comunidades no deben depender de ninguna clase de intereses ajenos a su finalidad específica, su compromiso debe circunscribirse al interés colectivo de sus integrantes, en particular. En cuanto a la influencia mutua, se destaca la participación como estrategia para contraer acuerdos, donde todos tengan la oportunidad de contribuir con sus opiniones a la toma de decisiones, lo cual genera la cooperación y el compromiso con las acciones a emprender. Sin duda, la interacción escuela-comunidad se puede lograr a través de la participación activa de los individuos en el proceso educativo, siendo parte en el mismo, tomando en cuenta la autogestión para que se pueda transformar su realidad. En la medida que se desarrolla este proceso continuo, el individuo va descubriendo, elaborando, inventando y enriqueciendo su conocimiento con su propia realidad. Hay un cambio de actitud hacia la crítica, los valores solidarios y comunitarios que van a depender del conocimiento que se tiene acerca de la participación, la multiculturalidad, la confianza, información, reconocimiento, el tiempo y el recurso. Para la ejecución de estrategias participativas en las escuelas se requiere que se establezcan metas, se diseñen políticas, se motive a los miembros o participantes y se asignen los recursos de manera que se puedan llevar a cabo en forma exitosa. 
Entre las formas o manifestaciones de la participación, según Casilla (2002), se encuentran los procesos de: toma de decisiones, conductas autónomas, racionalidad compartida, reflexión crítica, consenso, consulta, roles, responsabilidades, estructura, normas y evaluación, aprendizaje organizacionales, alcances y relaciones entre organizaciones, promoción e información transparente, estímulos, obstáculos y perturbaciones de la comunicación, jerarquías, productividad, competencias, autogestión y cogestión. Entre las estrategias teóricos-prácticas que favorecen la participación comunitaria en el hecho educativo y en la elaboración de proyectos que contribuyen con el bienestar individual, del colectivo y del ambiente está metodología participativa, que se presta al desarrollo de actividades para la preservación del medio ambiente.

\section{Metodología}

La metodología de la investigación respondió al objetivo determinar el nivel de participación de la comunidad en el PEIC, que aborda la educación ambiental en las instituciones de Educación Básica del Municipio Maracaibo, por tanto, se ubicó dentro del paradigma positivista, ya que se basa en observar los hechos para verificarlos. En cuanto a la metodología, el estudio se tipificó descriptivo, con diseño no experimental, ya que no se constituyó ninguna situación nueva, sino que se observaron las existentes para ser descritas. Se define también transversal dado que la recolección de datos, su análisis y descripción se hizo en un tiempo único, sin pretender estudiar la evolución de las variables en el tiempo. La población objeto de estudio estuvo constituida por un total de 924 individuos que fueron agrupados de acuerdo a su vinculación con las escuelas básicas seleccionadas, por tanto, participaron 3 directivos, 55 docentes, 17 obreros, 4 personal administrativo y 800 padres y representantes, con 45 sujetos miembros de la comunidad. Solo se calculó muestra para los padres y representantes quedando constituida por 89 sujetos.

Se elaboraron tres cuestionarios con cuatro alternativas siempre, casi siempre, casi nunca y nunca, uno dirigido a directores y docentes, otro para personal administrativo, obrero y padres y representantes, y el último para la comunidad, que fueron validados 
por expertos y cuya confiabilidad con la fórmula Alpha Cronbach dio $0,93,0,95$ y 0,92 respectivamente. La técnica de análisis fue la distribución de frecuencias, presentada en tablas con su respectivo análisis y discusión. Además, se construyó un baremo para interpretar los resultados acerca del nivel de participación de las comunidades escolares, determinando un nivel muy alto, si las respuestas de los encuestados se ubican en la alternativa siempre, alto si es casi siempre, un nivel moderado de participación cuando la selección es casi nunca y nunca indica que el nivel de participación es bajo tanto en las actividades de preservación del medio ambiente como en el Proyecto Educativo Integral Comunitario.

\section{Resultados de la investigación}

La aplicación de los cuestionarios a la población seleccionada: personal directivo, docente, personal administrativo y obrero, padres-representantes, y comunidad, reflejó su percepción acerca de la participación que tiene la comunidad educativa en general en actividades de preservación ambiental abordados en el PEIC, obteniendo los siguientes resultados:

\section{Tabla 1}

Indicador: Participación en actividades de preservación ambiental

\begin{tabular}{|c|c|c|c|c|c|c|c|c|c|}
\hline & \multicolumn{5}{|c|}{ Directivo-Docente } & \multicolumn{4}{|c|}{ Administrativo-Obrero } \\
\hline Alternativas & \multicolumn{5}{|c|}{ TOTAL } & \multicolumn{2}{|l|}{ Ítemes } & \multicolumn{2}{|r|}{ TOTAL } \\
\hline & & & & & Fr\% & & & $\mathrm{Fa}$ & Fr\% \\
\hline Siempre & 39 & 6 & 6 & 17 & 29,5 & 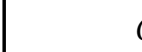 & 2 & 4 & 19,0 \\
\hline Casi siempre & 17 & 16 & 6 & 13 & 22,5 & 3 & 0 & 2 & 7,1 \\
\hline Casi nunca & 2 & 31 & 39 & 24 & 41,6 & 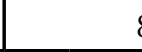 & 12 & 10 & 47,6 \\
\hline Nunca & 0 & 5 & 7 & 4 & 6,9 & 2 & 7 & 5 & 26,2 \\
\hline \multirow[t]{2}{*}{ Total } & 58 & 58 & 58 & 58 & 100 & 2 & 21 & 21 & 1100,0 \\
\hline & \multicolumn{5}{|c|}{ Padres y Representantes } & \multicolumn{3}{|c|}{ Comunidad } & \\
\hline Alternativas & \multicolumn{5}{|c|}{ TOTAL } & \multicolumn{4}{|r|}{ TOTAL } \\
\hline \multicolumn{6}{|c|}{$\mathrm{Fa}$} & \multicolumn{3}{|r|}{$\mathrm{Fa}$} & Fr\% \\
\hline Siempre & 48 & 12 & 4 & 21 & 24,0 & 0 & 0 & 0 & 0,0 \\
\hline
\end{tabular}


La educación ambiental en el proyecto educativo integral comunitaria: una reflexión desde la participación en comunidades escolares

\begin{tabular}{|l|r|r|r|r|r|r|r|r|r|r|}
\hline Casi siempre & 26 & 33 & 33 & 31 & 34,5 & 4 & 5 & 8 & 6 & 12,6 \\
\hline Casi nunca & 10 & 29 & 33 & 24 & 27,0 & 11 & 22 & 21 & 18 & 40,0 \\
\hline Nunca & 5 & 15 & 19 & 13 & 14,6 & 30 & 18 & 16 & 21 & 47,4 \\
\hline Total & 89 & 89 & 89 & 89 & 100,0 & 45 & 45 & 45 & 45 & 100,0 \\
\hline
\end{tabular}

Fuente: elaboración propia (2008)

De la misma forma, para el $47,6 \%$ del personal obrero-administrativo seguido por un $26,2 \%$ casi nunca y nunca los miembros de la comunidad tienen conocimiento de lo que se hace en la escuela, ni colaboran con la solución de problemas referidos al medio ambiente, sólo el 19\% siempre muestra interés al respecto, y un 7,1\% casi siempre. Para el caso de Padres y Representantes, el 34,5\% casi siempre participa en las actividades de la escuela, sobre preservación ambiental, pero poco intervienen en ellas, otro $27 \%$ casi nunca lo hace, pero un $24 \%$ casi siempre demuestra hacerlo y para el $14,6 \%$ nunca es así. Por su parte, en la comunidad externa, el 47,4\% nunca participa en las actividades de la escuela en referencia a este tipo de acciones, seguido por un $40 \%$ que casi nunca lo hace, sólo el 12,6\% casi siempre demuestra su participación en la institución.

Los resultados obtenidos se confrontaron con los autores que sustentaron las variables del presente estudio, por lo tanto se considera que en cuanto a la participación comunitaria en la solución de los problemas ambientales se observó que el personal directivo y docente siempre reconoce la importancia de la participación comunitaria, aceptando la acción de ellos, de allí que su actitud es positiva, lo cual es similar a lo expuesto por Chiavenato (2003) quien expresa que la persona refleja con ella lo que siente acerca de los demás, hecho que se constata cuando estos miembros del plantel se sienten bien con las actividades desarrolladas por la comunidad.

Tal posición fue coincidente con los resultados del personal administrativo y obrero del plantel así como de los padres, representantes y demás miembros de la comunidad, al considerar que la actitud asumida por el personal de éste es positiva abriendo posibilidades a la participación, que como lo expone Rivarosa (2005), es necesario dar a conocer la situación acerca de la educación ambiental en los proyectos educativos, buscando la intervención de distintos grupos humanos, coadyuvando a la 
conformación de una cultura ambientalista que contribuya con el desarrollo y fortalecimiento de la comunidad.

Sin embargo, se reflejó que los encuestados opinan que casi nunca se aprovechan los recursos humanos para realizar las actividades de preservación ambiental en la escuela, así como tampoco se buscan los recursos económicos, tecnológicos que ofrecen otras instituciones para llevar a cabo las acciones necesarias del plantel, por tanto, no se cuenta con el apoyo para la realización de éstos, lo cual diverge de lo expuesto por Viñas \& Doménech (1998), en cuanto al aprovechamiento efectivo de los recursos.

Los aspectos antes explicados evidenciaron que existen divergencias en las opiniones de los encuestados con respecto a la dimensión Participación en actividades de preservación ambiental, por cuanto para el personal directivo y docente, administrativo, obreros y comunidad en general, hay poca efectividad en cuanto a los valores, conocimiento, multiculturalidad, reconocimiento, tiempo y recursos, mientras los padres y representantes consideran que es moderadamente efectiva la actitud de todos hacia la participación en las actividades del plantel, que como expresa Casilla (2002), propicia la toma de decisiones compartida, la reflexión crítica y la responsabilidad por interactuar en estos proyectos ambientales.

Tabla 2

Indicador: Participación en el Proyecto Educativo Integral Comunitario

\begin{tabular}{|c|c|c|c|c|c|c|c|c|c|}
\hline & \multicolumn{5}{|c|}{ Directivo-Docente } & \multicolumn{4}{|c|}{ Administrativo-Obrero } \\
\hline \multirow[t]{2}{*}{ Alternativas } & & & & & TOTAL & \multicolumn{4}{|c|}{ TOTAL } \\
\hline & & & & $\mathrm{Fa}$ & Fr\% & & & $\mathrm{Fa}$ & $\mathrm{Fr} \%$ \\
\hline Siempre & 2 & 2 & 1 & 2 & 2,9 & 3 & 4 & 4 & 16,7 \\
\hline Casi siempre & 19 & 10 & 14 & 14 & 25 & 8 & 10 & 9 & 42,9 \\
\hline Casi nunca & 33 & 36 & 39 & 36 & 62 & 2 & 3 & 3 & 11,9 \\
\hline Nunca & 4 & 10 & 4 & 6 & 10 & 8 & 4 & 6 & 28,6 \\
\hline \multirow[t]{2}{*}{ Total } & 58 & 58 & 58 & 58 & 100 & 21 & 21 & 21 & 100 \\
\hline & \multicolumn{5}{|c|}{ Padres y Representantes } & \multicolumn{4}{|c|}{ Comunidad } \\
\hline \multirow[t]{2}{*}{ Alternativas } & & & & & TOTAL & \multicolumn{4}{|c|}{ TOTAL } \\
\hline & & & & $\overline{\mathrm{Fa}}$ & Fr\% & & & $\mathrm{Fa}$ & $\mathrm{Fr} \%$ \\
\hline
\end{tabular}


La educación ambiental en el proyecto educativo integral comunitaria: una reflexión desde la participación en comunidades escolares

\begin{tabular}{|l|r|r|r|r|r|r|r|r|r|r|}
\hline Siempre & 3 & 6 & 11 & 7 & 7,5 & 0 & 0 & 0 & 0 & 0,0 \\
\hline Casi siempre & 16 & 22 & 20 & 19 & 21,7 & 5 & 5 & 4 & 5 & 10,4 \\
\hline Casi nunca & 50 & 30 & 45 & 42 & 46,8 & 26 & 16 & 24 & 22 & 48,9 \\
\hline Nunca & 20 & 31 & 13 & 21 & 24,0 & 14 & 24 & 17 & 18 & 40,7 \\
\hline Total & 89 & 89 & 89 & 89 & 100,0 & 45 & 45 & 45 & 45 & 100,0 \\
\hline
\end{tabular}

Fuente: elaboración propia (2008)

En la tabla 2, con relación al indicador participación en el PEIC, como el $62 \%$ del personal directivo y docente, casi nunca involucra a la comunidad para la generación de ideas que contribuyan con los proyectos de la institución referidos a las actividades de preservación ambientalista, ni realiza investigaciones partiendo del conocimiento de las necesidades de la comunidad, así como tampoco toma en cuenta las experiencias de los miembros de la comunidad para el desarrollo de actividades que conduzcan a la solución de los problemas del medio ambiente; mientras otro $25 \%$ casi siempre lo hace así, para el 10\% nunca es así, y el 2,9\% restante siempre participa en este tipo de actividades. No obstante, el $42,9 \%$ del personal obrero-administrativo opina que casi siempre se involucra para dar solución a los problemas que enfrentan en la escuela y la comunidad, otro $28,6 \%$ nunca se involucra, el $16,7 \%$ siempre lo hace y un 11,9\% nunca. Para el caso de Padres y Representantes, el $46,8 \%$ casi nunca participa, junto a un $24 \%$ que nunca lo hace, otro $21,7 \%$ casi siempre lo hace y el $7,5 \%$ siempre. Por su parte, la comunidad externa, el $48,9 \%$ casi nunca se involucra en el PEIC, seguido por un 40,7\% que nunca lo hace, sólo el $10,4 \%$ lo hace casi siempre.

Como se observa, los resultados evidencian poca participación de directivos, padres, representantes y comunidad en el PEIC, que se programan desde la escuela, a través de las actividades planificadas por los docentes con sus estudiantes de educación Básica con respecto a la preservación del medio ambiente y desarrollar conciencia, contradiciendo lo expuesto por Carrasco (1996), al constatarse cierta resistencia o falta de información de estos actores en cuanto a las bondades de la investigación acción participativa que le permitirían asumir su compromiso con la construcción de ideas, 
y estrategias para el cambio, la preservación y la transformación de conocimientos y creencias acerca de la naturaleza.

Por lo tanto, al no involucrarse en los proyectos referidos a la educación ambiental desde la escuela para el bienestar de toda la comunidad, poco se está haciendo y como lo plantea Murcia (1992), no se está contribuyendo con el desarrollo ni con la superación de los conflictos y contradicciones del problema u objeto de estudio, por lo tanto, se manifiestan debilidades en la producción de conocimientos para guiar la práctica que conlleva a la modificación de una realidad dada como parte del mismo proceso investigativo en relación a la preservación del medio ambiente de la localidad donde conviven los ciudadanos. Cabe decir, que la participación detectada en la comunidad educativa con respecto al desarrollo de los Proyectos educativos, especialmente el PEIC, se encuentra en la escala de participación por consulta y por incentivo, lo cual explica lo expuesto por Geilfus (2005), es decir, se solicita opinión a los padres y representantes, ofreciéndole beneficios, pero no se estimula la responsabilidad y el compromiso, de allí que las actividades de Educación ambiental se realizan de manera pasiva, sin internalizar acerca de la importancia de preservar y conservar el medio ambiente.

\section{Consideraciones finales}

El análisis y reflexión de los resultados obtenidos en la presente investigación permiten concluir que la participación de la comunidad escolar en las actividades de preservación del medio ambiente en las escuelas básicas seleccionadas posee un nivel moderado, por cuanto para directivos, docentes, personal administrativo y obrero y la comunidad en general, casi nunca se involucran en éstas, aunque para los padres y representantes casi siempre se participa, lo cual demostró debilidad observando algunas divergencias en las opiniones aunque los patrones de diferenciación no son significativos para el estudio.

Con el estudio se pudo identificar que existen estrategias teóricas que desde la escuela favorecen la participación de la comunidad escolar pero realmente en la práctica éstas no se cumplen, aunque exista un soporte legal que ampara y exige la participación de la co- 
munidad educativa, la convivencia, comunicación, motivación y la investigación acción participativa como herramientas para lograr el cambio y la preservación del medio ambiente, a través de las cuales se evidencia que existen divergencias en las opiniones expresadas por todos los actores demostrando ausencia de interés colectivo en referencia a la participación en actividades de preservación ambientalista que permita la unificación de criterios y de acciones dirigidas a un punto común relacionado con la participación comunitaria.

La participación de las comunidades escolares abordadas en el PEIC, para el desarrollo de la educación ambiental, también se ve obstaculizada por el desconocimiento, concluyendo que, para directivos, docentes, padres-representantes y comunidad en general, tiene un nivel participación moderado, aunque el personal administrativo y obrero, manifestó que casi siempre se involucran en estos proyectos. De igual manera, al no darse una red comunicacional que trascienda a la escuela, llegando al ámbito comunitario, se establecen barreras en las relaciones escuela-comunidad, provocando desmotivación en la misma para la interpretación de los intereses socioculturales como estrategia para el logro de los objetivos deseados en cuanto a las interacciones que deben establecerse entre sociedad y naturaleza, que sustentan su intervención en los asuntos educativos y del medio ambiente, y a la vez normalizan la conducta de todos sus miembros a través de los consejos comunales.

Asimismo, se comprobó la opinión de directivos, docentes, personal administrativo y obrero en referencia a la moderada participación en el PEIC, en el desarrollo del trabajo en equipo de los miembros de la comunidad representando esto el desconocimiento del objetivo que se persigue y del futuro deseado para la escuela por parte de la misma., además que es una participación por consulta e incentivos. En tal sentido, la escuela no considera las necesidades prioritarias de su entorno comunal al planificar los proyectos pedagógicos, originando poco compromiso de parte de los miembros de la comunidad, así como tampoco se da la evaluación permanente del proceso educativo para la solución a los problemas de la escuela, que involucran a la comunidad, y viceversa.

Los aspectos antes expuestos, permiten concluir que al establecer el nivel de participación de la comunidad escolar en el PEIC, referido al desarrollo de la Educación ambiental en las escuelas bá- 
sicas seleccionadas en este estudio, éste resultó ser moderado según la percepción de los diferentes actores participantes de esta investigación, de allí que se comprueba lo expresado en el planteamiento del problema que dio origen al estudio en cuestión.

Al evidenciarse un nivel de participación moderado y poco efectivo por parte de la comunidad escolar en los proyectos para el desarrollo de la educación ambiental abordados en el PEIC, de la escuela, es pertinente expresar algunas recomendaciones como estrategias para propiciar el compromiso y la responsabilidad de todos los actores en la preservación del medio ambiente que los rodea y en la construcción de conocimientos donde se incluya la dimensión ambiental como punto clave del currículo y de los organismos comunales, enfatizando en la investigación para explorar, trabajar y proyectar las acciones de autogestión y cogestión conducente a la solución de problemas socio ambientales. Por lo tanto, se recomienda: desarrollar acciones para el acercamiento de los miembros de la comunidad, creando una red comunicacional que facilite el acceso a la información en el ámbito de la relación escuela-comunidad, desarrollando encuentros comunitarios en la sede, donde participen activamente todos en los proyectos de educación ambiental.

Brindar oportunidades a los miembros de la comunidad para que logren asumir responsabilidades que conduzcan al logro de los objetivos, promoviendo la lealtad en los miembros y el fomento de valores éticos, sociales, culturales y ambientalistas dentro del proceso educativo para el logro de la solución de la problemática ambiental, convirtiendo esto en un elemento clave de la visión establecida en la institución.

Tomar en cuenta lo que piensan y desean las personas para poderse adecuar a las exigencias del momento, de allí que en la medida que sea escuchada la opinión de los individuos, se sentirán involucrados, al relacionarse directamente con los hechos para sentir los riesgos de éste, valorando lo que hace y el grado de compromiso adquirido. Programar proyectos teórico prácticos desde el aula vinculados a la solución de la problemática ambiental partiendo de la localidad o región, propiciando la reflexión y construcción de conocimientos que sirvan para el debate y el desarrollo de competencias en los estudiantes, así como de los integrantes de la comunidad educativa. 
Constituir a la escuela en un espacio público que una los esfuerzos y voluntades de todos los actores sociales en procura de un marco de objetivos comunes; por lo tanto, se debe facilitar la escuela para realizar actividades dentro de la misma donde compartan y converjan ciudadanos, empresas, grupos de acción comunitaria (culturales, deportivas, vecinales, recreativas) desarrollando la solidaridad, la tolerancia, el respeto por las diferencia y la autonomía, dentro de un marco de convivencia armónica de los individuos con su medio ambiente.

Enriquecer la búsqueda de consenso y soluciones a los problemas ambientales, sin imposiciones, aunando los esfuerzos alcanzados en un fin común, aprovechando las legítimas diferencias ideológicas y políticas.

Establecer convenios o alianzas con personas e instituciones para apoyos diversos, no solo a nivel de mejorar el medio ambiente sino también para intervenir en el fortalecimiento de los proyectos de aprendizaje (charlas, películas, conferencias). Comprometer a las empresas que están alrededor de la escuela para que sirvan de espacio educativo con respecto a la formación del recurso humano que esté involucrado.

Desarrollar el PEIC, relacionado con Educación ambiental formal e informal, conjuntamente con todos los miembros de la Comunidad Educativa y las fuerzas vivas de la localidad, de manera que cumpla con todas sus funciones: pedagógica, administrativa, comunitaria y ambientalista. Involucrar a las instituciones formadoras (universidades, colegios universitarios) para que se desarrollen actividades educativas, sociales, culturales, entre otras, haciendo uso del espacio de la escuela y de la comunidad que la alberga.

Realizar encuentros entre las diversas instituciones educativas aledañas a la escuela para exponer experiencias y aportar ideas que sirvan de base para el desarrollo endógeno y exógeno de la comunidad. Promover la investigación-acción como medio para la búsqueda del conocimiento y la verdad, desarrollando a través de ella, un rol activo como promotores, facilitadores y orientadores del trabajo colectivo que involucra a todos los actores de la comunidad.

Dar respuestas a las exigencias y necesidades de la comunidad, atendiendo tanto los procesos como los resultados de la tarea educativa con miras al verdadero cambio, sin olvidar que lo más impor- 
tante son las personas y se aspira tengan calidad de vida, por ello, al desenvolverse en su medio ambiente, debe propiciarse una dinámica que permita satisfacer las necesidades y expectativas que poseen.

\section{Referencias bibliográficas}

Abreu, T (1996). Propuesta de una estrategia educativo ambiental basada en los principios del desarrollo sustentable y las características del visitante. Caso: Parque Recreacional Los Chorros. Trabajo de grado de maestría no publicado, Universidad Pedagógica Experimental Libertador, Instituto Pedagógico de Caracas, Caracas.

Alfiz, I. (2003). El proyecto educativo institucional. Argentina. Aique.

Ander, E. (2001). Planificación educativa. Conceptos, métodos, estrategias y técnicas para educadores. Buenos Aires: Editorial Magisterio Río de la Plata.

Bacalini, G. (1998). Haciendo Escuela: alternancia, Trabajo y Desarrollo En el Medio Rural. 1ºdición, Buenos Aires.

Benayas, J., Gutiérrez J. \& Hernández, N. (2003) La investigación en Educación ambiental en España. Ministerio del Medio ambiente. Secretaria General de Medio Ambiente. Organismo Autónomo. Parques Nacionales: Egraf, S.A.

Carrasco, M. (1996). La Educación Ambiental: una estrategia flexible, un proceso y unos propósitos en permanente construcción. La experiencia en Colombia. Revista Iberoamericana de Educación. Educación Ambiental y formación. OEI. No. 16.

Casilla, D. (2002). Tecnología Educativa para la participación comunitaria. Tesis doctoral. La Universidad del Zulia. Maracaibo.

Chiavenato I. (2003). Introducción a la teoría general de la administración. México: Mc Graw Hill.

Davis K. \& Newstrom J. (2004) Comportamiento humano en el trabajo. México: Mc Graw Hill.

Geilfus, F. (2005). 80 Herramientas para el desarrollo participativo. México:IICA: Holanda Laderas, C. A.

Ministerio de Educación, Cultura y Deportes. (2001). Proyecto Educativo Nacional. Caracas, Venezuela.

Morales, M. (2006). Orientaciones para la Construcción del Proyecto Educativo Integral Comunitario (PEIC). Quinta Versión. Ministerio de Educación y Deporte. Zona Educativa Zulia. División Académica. 
Pérez, A. (1999). Historia de una reforma educativa. España. Editorial Diada.

Rivarosa, A. (2005) Los proyectos escolares en Educación Ambiental: su potencial educativo y transformador. Disponible en:www. Ambiente.gov:ar/infontecala

Teitelbaum, A. (1978) El papel de la educación ambiental en América Latina. París: UNESCO

Santos M. (1998). El crisol de la participación. Estudio etnográfico sobre la participación en Consejos Escolares del Centro. España: Editorial Escuela Española.

Smith, N. (1997). Environmental Issues Information Sheet EI-publicada en University of Illinois Cooperative Extensión Service. http://www. nres.uiuc.edu/outreach/pubs/ei9709.pdf.)

Viñas J. \& Doménech J. (1998) El sistema relacional en organización de centros educativos. Aspectos básicos. Barcelona: Editorial Praxis. 